\title{
Antineoplastic Drugs as a Cause of Drug Induced- Immune Hemolytic Anemia, Resulting in Major Intravascular Hemolysis Leading to Acute Kidney Injury Requiring Renal Replacement Therapy
}

\section{Pierre Bay}

Sorbonne Université, Assistance Publique-Hôpitaux de Paris (APHP), Hôpital Tenon, Soins intensifs néphrologiques et rein aigu

\section{David Buob}

Sorbonne Université, Assistance Publique-Hôpitaux de Paris (APHP), Hôpital Tenon

\section{Paul Gabarre}

Sorbonne Université, Assistance Publique-Hôpitaux de Paris (APHP), Hôpital Saint Antoine, Réanimation Médicale

\section{Eric Rondeau}

Sorbonne Université, Assistance Publique-Hôpitaux de Paris (APHP), Hôpital Tenon, Soins intensifs néphrologiques et rein aigu

\section{Julie Fillon}

Sorbonne Université, Assistance Publique-Hôpitaux de Paris (APHP), Hôpital Tenon

\section{Jean-Baptiste Gibier}

CHU Lille

\section{Yosu Luque}

Sorbonne Université, Assistance Publique-Hôpitaux de Paris (APHP), Hôpital Tenon, Soins intensifs néphrologiques et rein aigu

Thomas Modot

Etablissement Français du Sang, Ile de France, Hôpital Saint Antoine, Paris France.

\section{Alice Doreille}

Sorbonne Université, Assistance Publique-Hôpitaux de Paris (APHP), Hôpital Tenon, Soins intensifs néphrologiques et rein aigu

\section{Cedric Rafat ( $\nabla$ cedric.rafat@aphp.fr)}

Sorbonne Université, Assistance Publique-Hôpitaux de Paris (APHP), Hôpital Tenon, Soins intensifs néphrologiques et rein aigu

\section{Research Article}


Keywords: antineoplastic drugs, immune hemolysis, thrombocytopenia, acute kidney injury, renal replacement therapy

Posted Date: January 28th, 2022

DOI: https://doi.org/10.21203/rs.3.rs-1068704/v1

License: (c) (i) This work is licensed under a Creative Commons Attribution 4.0 International License. Read Full License 


\section{Abstract \\ Background}

Drug-induced immune hemolysis (DIIHA) is rare and may involve antineoplastic agent. Life threatening DIIHA complicated with severe acute kidney injury (AKI) has hitherto seldom been reported and had yet to be scrutinized from a clinical standpoint.

\section{Methods}

Over a five-month period we admitted in our intensive care unit three patients with KDIGO stage $3 \mathrm{AKI}$ along with, hemolytic anemia and thrombocytopenia within 24-hours following the administration of antineoplastic agent. These three women underwent anti-neoplastic treatment for metastatic colorectal cancer.

\section{Results}

All three patients presented with 1) abrupt immune intravascular hemolysis along with thrombocytopenia 2) a positive direct antiglobulin test (DAT) positive for IgG 3) absence of schistocytes 4) and AKI requiring replacement therapy. All patients had multiple previous exposure to the drugs received. Kidney biopsy was performed for patient 1 , displaying acute tubular necrosis lesions interpreted as pigment nephropathy. Investigations in patients 2 and 3 highlighted high-titers of specific oxaliplatin-dependent antibodies. Hemolysis resolved within 14 days post-chemotherapy for all three patients. The renal outcome was favorable.

\section{Conclusions}

Antineoplastic drugs can elicit abrupt immune hemolysis and thrombocytopenia, resulting in severe AKI. Thorough explorations are warranted to confirm DIHA, incriminate the offending drug on proper grounds while exonerating other pharmacological therapies, a chief concern in the setting of cancer therapy so not to needlessly reduce anti-cancer treatment options. The outlook for renal recovery seems favorable.

\section{Introduction}

Nephrotoxicity arising from chemotherapeutic regimen represents one of the chief attributes of a new field termed onconephrology dedicated to the study of the relationship between cancer and renal disease (1). The comprehensive scrutiny of the mechanisms underpinning acute kidney injury (AKI) in the setting of cancer is all the more crucial as it represents a prominent complication, known to portend an ominous prognosis and to jeopardize therapeutic options. The incidence of malignancy-related AKI is estimated to range between 14 and $20 \%(1,2)$, reaching rates as high as $66.5 \%$ in specific settings $(1,3)$. One of the 
key challenges a clinician faces when confronted with cancer-related AKI is to determine whether chemotherapy is involved rather than the cancer per se, and subsequently to decipher the physiopathological connection between the treatment and renal disease, kept in mind that the patient is often exposed to a myriad of nephrotoxic therapies. Failing to establish the causal link between the offending chemotherapeutic drug and AKI may result in severe systemic manifestations due to chemotoxicity and, conversely, wrongly incriminating a drug may mislead clinicians in discarding a potentially life-saving treatment.

Drug-induced intravascular hemolysis (DIIHA) stands out as an exceptional (estimated incidence of 1 case per million per year) but potentially life-threatening condition complicating the use of antineoplastic drugs (4). Kidneys are known to be especially vulnerable to the toxic effects of free hemoglobin and, accordingly, AKI ranks among the chief concerns associated with IVH (5). Herein, we report three cases of DIIHA remarkable for sharing common clinical features and encapsulate the challenges when investigating and managing AKI in the setting of cancer.

\section{Material And Methods}

Over a five months period we admitted in our intensive care unit three patients with KDIGO stage 3 AKI along with, hemolytic anemia and thrombocytopenia within 24-hours following the administration of antineoplastic agent. We retrospectively describe the clinical and laboratory characteristics and the outcome of these three patients. Direct anti globulin testing was performed to ascertain auto-immune hemolysis. In addition, oxaliplatin-specific autoantibodies were sought for using the following technique: microcolumns containing gel and polyspecific anti-human globulin IgG + C3d (DG Gel Coombs Card, Diagnostic Grifols, Parets del Vallès, Spain) were utilized. Compatible normal donor red blood cells (RBCs) were prepared in $\mathrm{NaCl} 0.15 \mathrm{M}$ and suspended to $1 \%$. Patient's serum and a pool of normal donor $A B$ sera (control) were tested against untreated and/or enzyme (trypsin)-treated compatible normal donor RBCs. In each respective gel microcolumn, $50 \mu$ of cell suspension was added to the upper chamber and $25 \mu$ l of patient's serum was added to the RBCs. For drug testing, either $25 \mu$ of oxaliplatin or PBS (control) was added to the microcolumns. The columns were incubated for 1 hour at $37 \circ \mathrm{C}$ and thereafter submitted to centrifugation. Results were read by visual inspection and reactions were graded according to manufacturer's instructions. Positive results were recorded on a scale ranging from very weak $(0.5+)$ to very strong (4+) (figure 1) (6).

Immunohistochemistry against haemoglobin (Recombinant Anti-Hemoglobin subunit alpha antibody, ref ab92492, Abcam, Paris, France) performed on paraffin-embedded sections of the available kidney biopsy specimen.

\section{Results}

These three female patients (aged 39, 44 and 75) were subjected to anti-neoplastic therapy for metastatic colorectal adenocarcinoma. Baseline kidney function was normal at baseline in each case and patients 
were free from other risk factors for renal disease (Figure 2 and 3).

Patient 1 (aged 44) had a history of multiple lines of anti-neoplastic treatment for sigmoid colon adenocarcinoma for the last 3 years, with initial exposure to irinotecan as part of first line chemotherapy (combination of 5-FU, irinotecan and oxaliplatin). Tumor progression subsequently required several lines of treatment with 5-FU, oxaliplatin and cetuximab (anti-EGFR). Prior to admission to the nephrology department, she had received a combination therapy with 5-FU, irinotecan and aflibercept (anti-VEGF) owing to disease progression as manifested by an enlarged nodule seated in the liver. Within 48 hours of administration of the first course of the new anti-neoplastic regimen she developed abrupt immune IVH: lactate dehydrogenase (LDH) levels peaked at $2258 \mathrm{UI} / \mathrm{L}$ with platelets levels reaching a nadir level of 58000 platelets $/ \mathrm{mm}^{3}$ followed, within hours, by AKI KDIGO III requiring renal replacement therapy (RRT) for eight days. Clinical examination and ultrasound examination of the heart and urinary tract were unremarkable. The direct antiglobulin test (DAT) was positive for IgG and C3d. No schistocyte was found. A kidney biopsy was performed; the biopsy specimen contained 19 glomeruli including one sclerotic glomerulus. The pathological examination disclosed evidence for widespread acute tubular injury. Intratubular eosinophilic casts were noticed, some with a "beadlike" morphology. Prussian blue stain did not detect hemosiderin deposits within the renal tissue whereas immunohistochemistry with an antihemoglobin antibody showed several positive casts (figure 4). No evidence for TMA was found.

Patient 2 (aged 39) had a history remarkable for colic adenocarcinoma with peritoneal dissemination which required surgical debulking and multiple lines of chemotherapy over the last 5 years, with multiple exposures to oxaliplatin. Two years later, she presented a disease progression requiring a new treatment combining 5-FU, oxaliplatin and Bevacizumab (anti VEGF). The persistence of peritoneal carcinosis prompted a treatment combining 5-FU, panitumumab (anti-EGFR agent), and two rounds of oxaliplatinbased pressurised intraperitoneal aerosol chemotherapy (PIPAC) 6 weeks apart. Within 24 hours of administration of second course of PIPAC she developed abrupt immune IVH with stage 3 KDIGO AKI. Blood pressure was normal and screening for sepsis was negative. Heart and urinary tract ultrasound examination was normal. LDH achieved peak levels of $3000 \mathrm{UI} / \mathrm{L}$ while hemoglobin and platelets plummeted to $4 \mathrm{~g} / \mathrm{dL}$ and $18000 / \mathrm{mm}^{3}$. The direct antiglobulin test (DAT) was positive for lgG. Schistocytes were absent. Bone marrow aspiration showed normal hematopoietic populations free from tumoral infiltration. Investigations highlighted high-titers of specific oxaliplatin-dependent antibodies with similar results for other cisplatin and carboplatin. No specific 5-FU-dependant antibodies were evidenced.

Patient 3 (aged 75) had undergone surgery and received adjuvant oxaliplatin therapy (6 rounds) for colorectal adenocarcinoma five years earlier. Peritoneal dissemination to the peritoneum prompted a new line of treatment comprising 5-FU, oxaliplatin and panitumumab. Within 24 hours of third administration, she developed, abrupt immune IVH with concurrent thrombocytopenia and AKI KDIGO III occurred within 6 hours. She required RRT for three days. Heart and urinary tract ultrasound investigations were normal. The lowest recorded platelet level reached $76000 / \mathrm{mm}^{3}$ and LDH attained a peak level of 2020 UI/L. The direct antiglobulin test (DAT) was positive for lgG. Schistocytes were absent. Investigations highlighted 
high-titers of specific oxaliplatin-dependent antibodies. No specific 5-FU-dependant antibodies were evidence.

Patients 1 and 2 were treated with a short bout of corticosteroid therapy. Hemolysis resolved after 10 to 14 days in all three cases. The renal outcome was favorable: patient 1 and 2 exhibited a complete recovery of renal function within two months, while patient 3 displayed partial recovery with an eGFR of $49 \mathrm{~mL} / \mathrm{min} / 1,73 \mathrm{~m}^{2}$ (CKD-EPI) at three months upon follow-up.

\section{Discussion}

All three patients presented with 1) abrupt and severe immune intravascular hemolysis along with thrombocytopenia, 2) a positive direct antiglobulin test (DAT) positive for IgG in all three patients and positive for C3d in patient 1,3 ) thrombotic microangiopathy (TMA) was ruled out, based on absent schizocytes (patients 1,2 and 3), negative blood marrow investigation (patient $X$ ) and renal pathology (patient Y) 4) and AKI requiring replacement therapy within 24 hours after administration of antineoplastic therapy.

The renal toxicity of hemolysis is ascribed to the release of hemoproteins (hemoglobin and heme) due to the breakdown of erythrocytes. The heme group harbours an iron atom in the ferrous state, which exhibits an exquisite affinity or nitric oxide (NO) and acts a potent inductor of redox reactions. Severe NO depletion ensues resulting in vasoconstriction altered renal perfusion (7). The pro-oxydative state in turn unleashes lipid peroxidation and elicits reactive oxygen species-associated cytotoxicity following glomerular filtration of heme, and reabsorption in the proximal tubule (8). Cell-free heme activation of the complement and tubular obstruction by hemoglobin casts may also precipitate AKI (8). A multicomponent detoxification system - in the heart of which lies haptoglobin and hemopexin- plays a pivotal role, aptly counteracts the nefarious effects of the cell free hemoglobin/heme proteins (9). This system alleviates the consequences of mild to moderate haemolysis, but may be exhausted if extensive and/or persistent hemolysis occurs, in which case hemopexin steps in acting as a rescue pathway (9). However, in case of massive IVH the overflow of hemoglobin/heme is thought to overwhelm resulting in AKI. In addition, hemolysis may generate AKI via hemoglobin cast-induced tubular obstruction. From this standpoint, the histopathological examination of the kidney provided further insights into what are the relevant mechanisms responsible for the renal toxicity of IVH. The absence of cytoplasmic hemosiderin deposits in tubular cells ("pigment nephropathy") pleaded against direct heme toxicity of the proximal tubular epithelium (patient 1). The finding of hemoglobin-positive casts suggests the possible role of tubular obstruction.

Oxaliplatin is a third generation platinum derivative and a mainstay anti-neoplastic drug as part of the first line of colorectal chemotherapy (10). Oxaliplatin has been known to induce hypersensitivity reaction within minutes or hours of completing the drug's infusion and following multiple cycles of treatment suggesting the importance of prior sensitization as highlighted in patients 2 and 3 (11). The drug has risen to become one of the leading pharmacological agents most commonly responsible for DIIHA. 
Patients with oxaliplatin-associated DIIHA typically exhibit positive DAT with anti-IgG and/or anti-C3 specificity $(12,13)$. Two competing immunopathological theories have been devised for DIIHA. First, the drug may elicit an antibody-mediated reaction either directed against the drug or after it binds the erythrocyte surface (i.e hapten mechanism). Second, the emergence of a neoantigen may arise from the combination of the drug and some components of the erythrocytes thereafter triggering the auto immune response (i.e neo-antigen mechanism) $(4,14)$. Alternatively, a non-immunologic hypothesis postulates that the offending drug may alter the erythrocyte's cell membrane leading his spleen destruction by macrophage-related phagocytosis (15). Importantly, positive DAT tests do not discriminate between both mechanisms and in the case of oxaliplatin both concepts have been purported and may in fact well coexist (13). The thrombocytopenia seems to closely relate to DIIHA in terms of underlying pathogenesis, going so far as to serve as a conceptual framework for DIAlHA (14). Thrombocytopenia was in fact a consistent feature across all three cases. Investigators may take advantage of these concepts to seek drug specific hemolysis by admixing the patient's sera with blood group-matched erythrocytes along with the suspected drug and in the presence of polyspecific lgG and the C3 complement fraction. In two cases, assays based on this model unraveled oxaliplatin-specific anti-erythrocyte autoantibodies and further enabled to predict the risk of DIIHA in case of exposure to other platinum-derived compounds. In the case of patient 1 , drug specific assays were not performed so that we could not adjudicate conclusively whether 5-FU, aflibercept or irinotecan was involved. Based on clinical considerations, irinotecan was suspected as the offending drug. In line with this 1) she later received 5-FU treatments without complication and 2) she had never been previously exposed to aflibercept, a fact inconsistent with the pre-sensitization condition.

PIPAC has recently emerged as a welcome innovation in the face of peritoneal carcinomatosis. This strategy hinges on the concept of enhancing local delivery of antineoplastic agents while at the same time safeguarding patients from systemic chemotoxicity. Studies have previously shown that patients exposed to cisplatin-based PIPAC did not manifest evidence of renal toxicity (16). At variance, case 2 demonstrates instead that AKI remains a possible occurrence at least through DIIHA-related mechanism and regardless of the level of drug plasma concentration.

\section{Conclusion}

All three cases bear witness to the severity of DIIHA-related IVH as manifested by abrupt hemolysis and KDIGO stage III AKI. Thrombocytopenia appears to be a salient and potentially misleading feature. Thorough explorations are warranted to confirm DIIHA, incriminate the offending drug on proper grounds while exonerating other pharmacological agents, a chief concern in the setting of cancer therapy so not to needlessly reduce anti-cancer treatment options. The outlook for renal recovery seems favorable.

\section{Abbreviations}

AKI

acute kidney injury 
DAT

Direct Antiglobulin Test

DIIHA

Drug-induced immune hemolytic anemia

IVH

intra vascular hemolysis

LDH

lactate dehydrogenase

PIPAC

pressurised intraperitoneal aerosol chemotherapy

RBC

red blood cells

RRT

renal replacement therapy

TMA

thrombotic microangiopathy

\section{Declarations}

ETHICS APPROVAL AND CONSENT TO PARTICIPATE: The database is registered with the "Commission Nationale de l'Informatique et des Libertés" (n²223905). In accordance with French Law relating to the processing of personal data in the context of research not involving the human person (Délibération $\mathrm{n}^{\circ}$ 2018-155 du 3 mai 2018, JORF du 13 juillet 2018), the hospital's institutional review board was deemed unnecessary. Written informed consent was not needed because this observational study did not modify existing diagnostic or therapeutic strategies; however, patients were informed of their inclusion in the study.

CONSENT FOR PUBLICATION: Not applicable

AVAILABILITY OF DATA AND MATERIALS: The datasets used and/or analysed during the current study are available from the corresponding author on reasonable request.

All methods were performed in accordance with the relevant guidelines and regulations

COMPETING INTERESTS: none

FUNDING: none

\section{AUTHORS CONTRIBUTION:}

Pierre Bay, Cedric Rafat and Alice Doreille designed the study and analysed the data. Pierre Bay, Cedric Rafat and Alice Doreille wrote the manuscript. David Buob, Thomas Modot, Julie Fillon and Jean-Baptiste Gibier contributed data. All authors discussed the results and contributed to the final manuscript. 


\section{References}

1. Salahudeen AK, Bonventre JV. Onconephrology: the latest frontier in the war against kidney disease. J Am Soc Nephrol. 2013 Jan;24(1):26-30.

2. Jin J, Wang Y, Shen Q, Gong J, Zhao L, He Q. Acute kidney injury in cancer patients: A nationwide survey in China. Sci Rep. 2019 Mar 5;9(1):3540.

3. Darmon $M$, Vincent $F$, Canet $E$, Mokart $D$, Pène $F$, Kouatchet $A$, et al. Acute kidney injury in critically ill patients with haematological malignancies: results of a multicentre cohort study from the Groupe de Recherche en Réanimation Respiratoire en Onco-Hématologie. Nephrol Dial Transplant. 2015 Dec;30(12):2006-13.

4. Garratty G. Immune hemolytic anemia associated with drug therapy. Blood Rev. 2010 Sep;24(45):143-50.

5. Dvanajscak Z, Walker PD, Cossey LN, Messias NC, Boils CL, Kuperman MB, et al. Hemolysisassociated hemoglobin cast nephropathy results from a range of clinicopathologic disorders. Kidney Int. 2019 Dec;96(6):1400-7.

6. Nguyen TN, Fihman V, Maenulein E, Vinatier I, Klaren JM. Drug-induced immune hemolytic anemia investigation: Comparison between tube test and microcolumn agglutination (gel test) for the detection of drug-dependent antibodies in the presence of soluble drug. Transfus Clin Biol. 2020 Aug;27(3):133-8.

7. Garratty G. Drug-induced immune hemolytic anemia. Hematology Am Soc Hematol Educ Program. 2009;73-9.

8. Boutaud O, Moore KP, Reeder BJ, Harry D, Howie AJ, Wang S, et al. Acetaminophen inhibits hemoprotein-catalyzed lipid peroxidation and attenuates rhabdomyolysis-induced renal failure. Proc Natl Acad Sci U S A. 2010 Feb 9;107(6):2699-704.

9. Schaer DJ, Vinchi F, Ingoglia G, Tolosano E, Buehler PW. Haptoglobin, hemopexin, and related defense pathways-basic science, clinical perspectives, and drug development. Front Physiol. 2014;5:415.

10. André T, Boni C, Mounedji-Boudiaf L, Navarro M, Tabernero J, Hickish T, et al. Oxaliplatin, fluorouracil, and leucovorin as adjuvant treatment for colon cancer. N Engl J Med. 2004 Jun 3;350(23):2343-51.

11. Hewitt MR, Sun W. Oxaliplatin-associated hypersensitivity reactions: clinical presentation and management. Clin Colorectal Cancer. 2006 Jul;6(2):114-7.

12. Nguyen TN, Maenulein E, Fihman V, Vinatier I, Moh Klaren J. Serologic characteristics of oxaliplatin antibodies in 15 patients with drug-induced immune hemolytic anemia. Transfusion. 2021 May;61(5):1609-16.

13. Arndt P, Garratty G, Isaak E, Bolger M, Lu Q. Positive direct and indirect antiglobulin tests associated with oxaliplatin can be due to drug antibody and/or drug-induced nonimmunologic protein 
adsorption. Transfusion. 2009 Apr;49(4):711-8.

14. Rousseau C, Nguyen TN, Rebibou JM, Bastie JN, Audia S, Darut-Jouve A, et al. Oxaliplatin-Induced Evans Syndrome: A Possible Dual Mechanism. Clin Colorectal Cancer. 2020 Mar;19(1):57-60.

15. Garratty G. Immune hemolytic anemia caused by drugs. Expert Opin Drug Saf. 2012 Jul;11(4):63542.

16. Alyami M, Hübner M, Grass F, Bakrin N, Villeneuve L, Laplace N, et al. Pressurised intraperitoneal aerosol chemotherapy: rationale, evidence, and potential indications. Lancet Oncol. 2019 Jul;20(7):e368-77.

\section{Figures}


RBC from blood group matching individual ( $1 \%$ suspension)

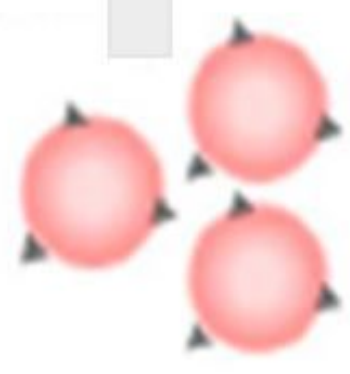

$50 \mu \mathrm{l}$

\author{
Patient sera
}

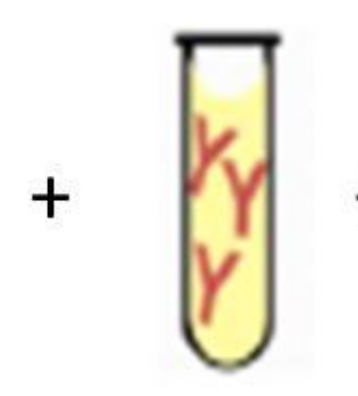

$+\quad$ Platinum drug

$25 \mu \mathrm{l}$

$25 \mu \mathrm{l}$
Upper chamber of a microcolumn with gel and polyspecific AHG IgG + C3d

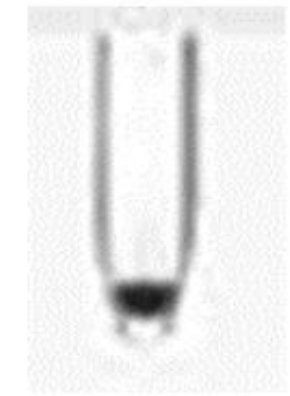

Negative

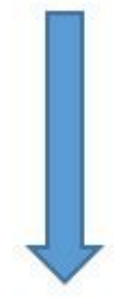

$$
\begin{aligned}
& -1 \mathrm{~h} / 37^{\circ} \mathrm{C} \\
& \text { - Centrifugation }
\end{aligned}
$$

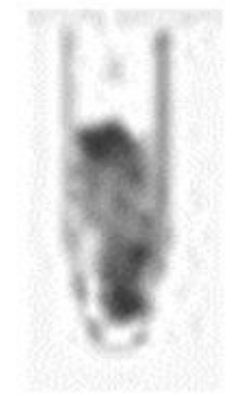

Positive

\section{Figure 1}

Schematic illustration of the procedure used to determine the cause of hemolysis 


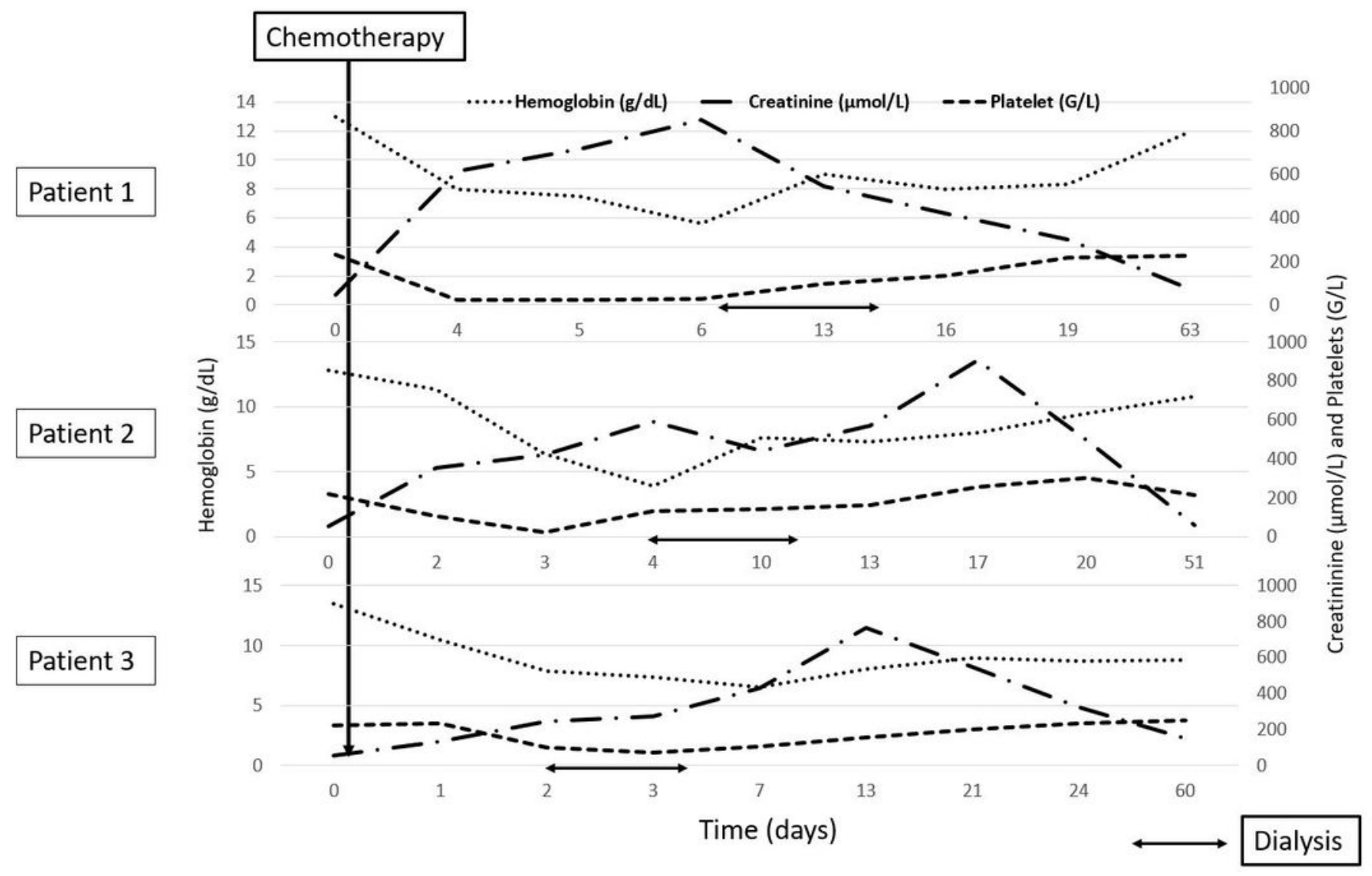

Figure 2

Anti-neoplastic history of the three patients

Previous oxaliplatin-based chemotherapy こニニニニト Chemotherapy including irinotecan
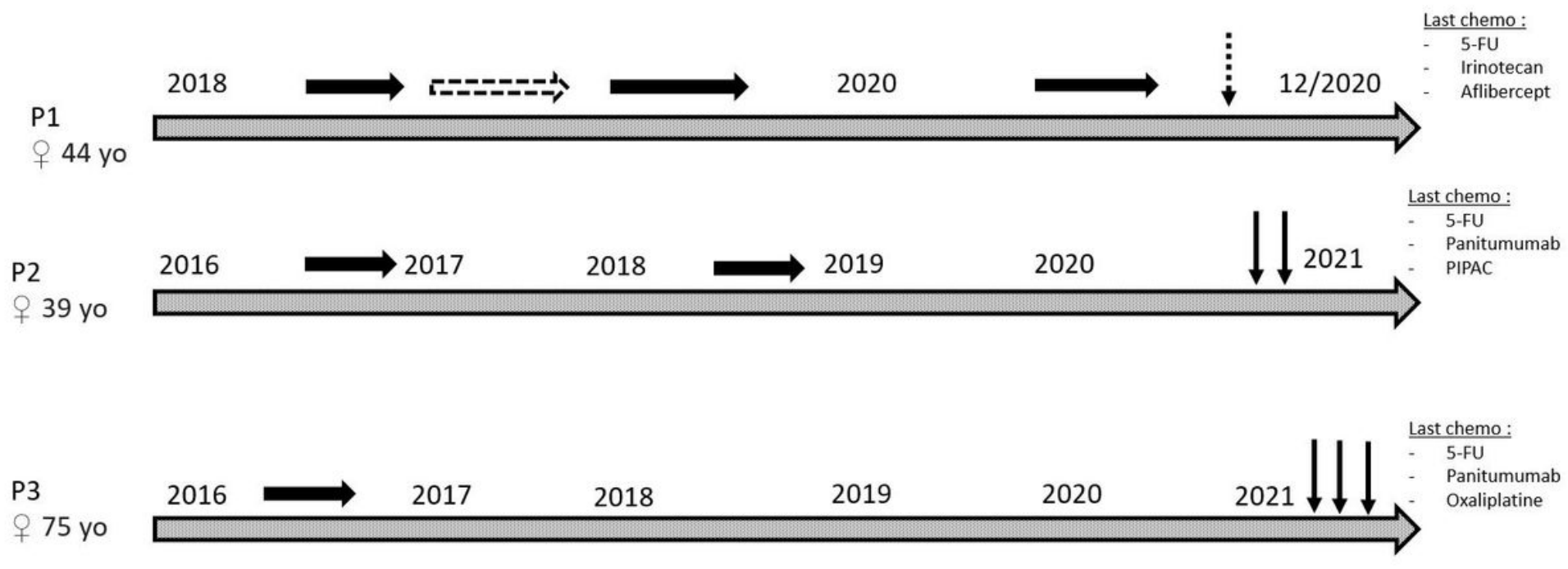
Figure 3

Laboratory findings and course of the three patients.
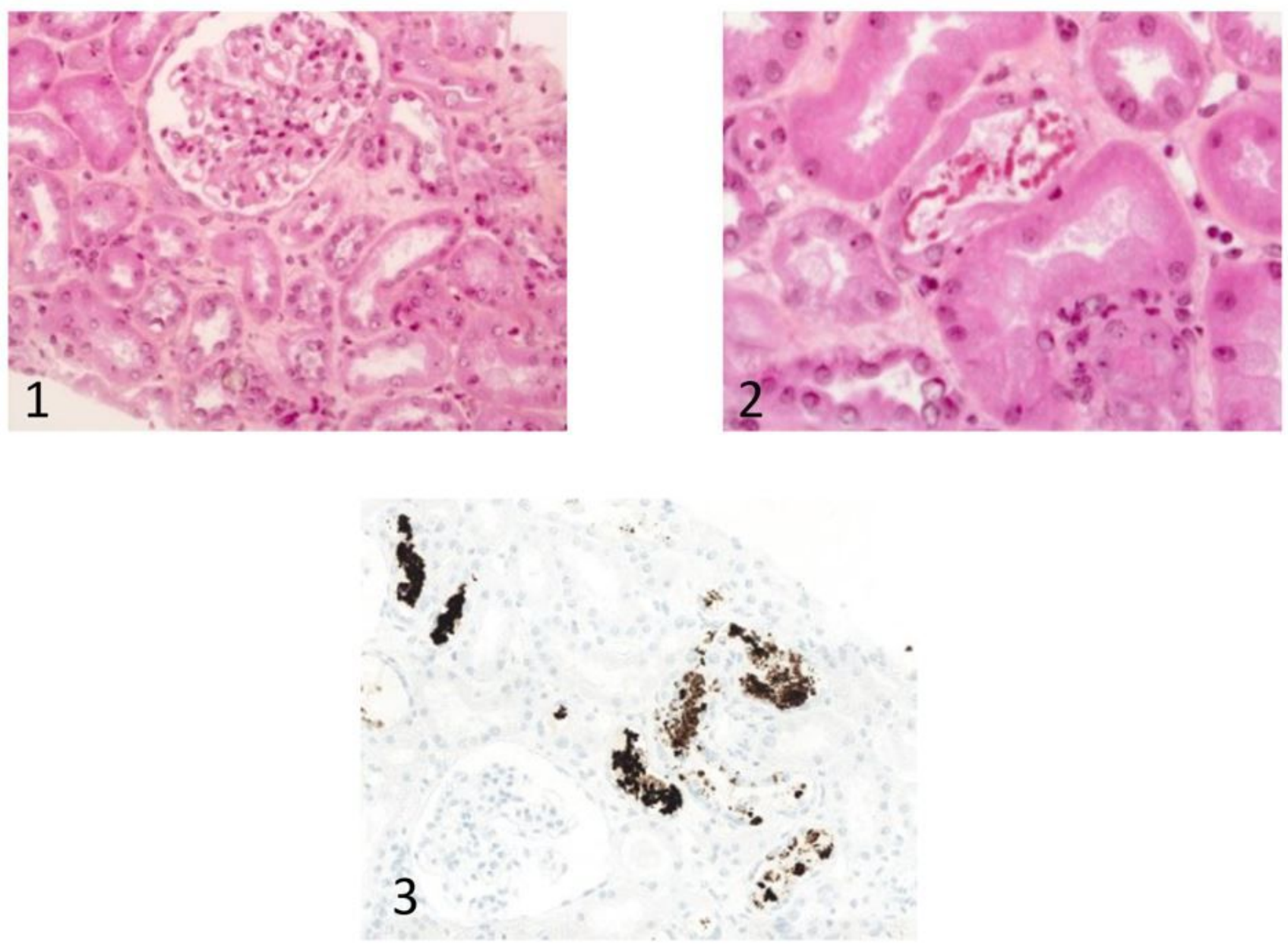

\section{Figure 4}

Histology findings of patient 1. Photo 1: acute tubular injury. Photo 2: intratubular casts with beadlike morphology. Photo 3: staining of several casts with anti-hemoglobin antibody. 